Meta

Journal des traducteurs

Translators' Journal

\title{
Des lexiques en langues africaines (sängö, wolof, lingála) pour l'utilisateur de l'ordinateur
}

\section{Marcel Diki-Kidiri, Chérif Mbodj et Atibakwa Baboya Edema}

Volume 42, numéro 1, mars 1997

Lexicologie et terminologie

URI : https://id.erudit.org/iderudit/003313ar

DOI : https://doi.org/10.7202/003313ar

Aller au sommaire du numéro

Éditeur(s)

Les Presses de l'Université de Montréal

ISSN

0026-0452 (imprimé)

1492-1421 (numérique)

Découvrir la revue

Citer cet article

Diki-Kidiri, M., Mbodj, C. \& Edema, A. (1997). Des lexiques en langues africaines (sängö, wolof, lingála) pour l'utilisateur de l'ordinateur. Meta, 42(1), 94-109. https://doi.org/10.7202/003313ar
Résumé de l'article

Les auteurs s'intéressent ici à l'expression d'un même savoir (utilisation de l'ordinateur) dans des langues et des cultures différentes, en analysant minutieusement les processus de conceptualisation mis en œuvre dans la langue de chaque culture. Cela leur permet d'aborder a) des questions techniques relatives à la terminographie en langues africaines; b) les exigences de l'utilisateur par rapport à la néologie et à la langue de spécialité ; c) la question du support : lexique hypermedia versus lexique sur papier. 


\title{
DES LEXIQUES EN LANGUES AFRICAINES (SÄNGÖ, WOLOF, LINGÁLA) POUR L'UTILISATEUR DE L'ORDINATEUR*
}

\author{
Marcel Diki-Kidiri, ChÉrif Mbodj et AtibakWa Baboya Edema \\ LLACAN (CNRS), Paris, France, CLAD, Université Cheikh Anta Diop, \\ Dakar, Sénégal et LLACAN / CELTA, Kinshasa, Gombé, Zaïre
}

\begin{abstract}
Résumé
Les auteurs s' intéressent ici à l'expression d'un même savoir (utilisation de l'ordinateur) dans des langues et des cultures différentes, en analysant minutieusement les processus de conceptualisation mis en auvre dans la langue de chaque culture. Cela leur permet d'aborder a) des questions techniques relatives à la terminographie en langues africaines; b) les exigences de l'utilisateur par rapport à la néologie et à la langue de spécialité ; c) la question du support: lexique hypermédia versus lexique sur papier.
\end{abstract}

\begin{abstract}
The authors look at how to express computer-related concepts in different languages and cultures. Through minutely detailed analysis of the conceptualisation processes at work, they discuss: 1) technical terminology problems in African languages; b) user requirements in terms of neology and specialized language; c) technical support problems: hypermedia lexicon versus lexicon on paper.
\end{abstract}

\section{PROBlÉMATIQUE}

Lors du premier séminaire des linguistes du Zaïre tenu à Lubumbashi en 1974, des étudiants de philologie «s'amusèrent» à traduire qui Platon, qui Horace, qui saint Thomas d'Aquin, qui le matérialisme de Karl Marx, qui la physique atomique, qui le marxisme et l'existentialisme en diverses langues zaïroises : swahili, lingála, cilùba, kikoongo, kiyaka, songye et yansi.

Le promoteur de cet exercice, un professeur de lettres, voulait montrer par là que «techniquement, nos langues [...] sont parfaitement capables de supporter et d'assumer n'importe quelle pensée» (CELTA 1974:22). Ayant intitulé son texte «une linguistique au service de l'homme», l'auteur voulait aussi démontrer qu'en réalité, la promotion des langues zaïroises relevait moins d'une compétence technique interne à la langue que d'une volonté des instances politiques officielles.

On se souviendra également qu'au Sénégal, Cheik Anta Diop a publié, en 1975, un article intitulé «Comment enraciner la science en Afrique...». Dans cet article (Diop 1975: 154-233), il propose un ensemble de traductions en wolof dans plusieurs domaines scientifiques tels que :

- la théorie des ensembles;

- la physique mathématique et théorique ;

- l'organisation de la matière au niveau subquantique et quantique ;

- la relativité restreinte et générale;

- la cosmologie relativiste;

- la terminologie de l'algèbre sensorielle;

- la chimie, etc.

Meta, XLLI. 1, 1997 
Cheik Anta Diop a ainsi permis à bon nombre de Sénégalais de se rendre compte de la capacité de leurs langues maternelles à s'adapter à toutes les exigences de la vie moderne.

Aujourd'hui, on en est plus à démontrer la capacité intrinsèque des langues, quelles qu'elles soient, à exprimer des concepts nouveaux. Cette capacité d'adaptation est, en réalité, une propriété intrinsèque de toute langue, tout simplement parce que inhérente à l'espèce humaine. Les langues sont autant des produits culturels que des instruments de façonnage de la culture qui les porte. Dès lors, la seule question fondamentale qui nous importe ici est de savoir comment les langues, et en particulier les langues africaines pour ce qui nous concerne, peuvent être exploitées par leurs locuteurs pour appréhender la nouveauté, la décrire, la conceptualiser et finalement l'assimiler dans leurs cultures propres.

Le développement fulgurant des technologies de l'information, tout comme la mondialisation grandissante des échanges économiques, font que des technologies de pointe, ou tout au moins certains produits de ces technologies, atteignent de plus en plus rapidement les communautés humaines les moins outillées, notamment celles d'Afrique. Imaginons alors la situation suivante comme un cas d'école.

Un chef de service africain, ayant bénéficié d'un stage de formation en bureautique à l'étranger, doit former à son tour toutes les secrétaires de l'institution où il travaille. Mais plusieurs de ces indispensables personnes maîtrisent mal le français et pas du tout l'anglais. De plus, n'ayant jamais travaillé autrement qu'avec de vieilles machines à écrire mécaniques et des méthodes antédiluviennes, elles éprouvent une véritable angoisse devant la perspective de se mettre à l'ordinateur, avec tout le bouleversement que cela implique. La direction décide de tenter l'expérience d'une formation donnée dans la langue africaine commune à tous. La question est de savoir comment rendre dans la langue africaine en question tous les concepts inédits qui entrent en jeu dès l'utilisation d'un ordinateur. Heureusement pour la direction, des linguistes, des terminologues, des aménageurs langagiers ont étudié la question et préparé, avec le concours des utilisateurs, des lexiques spécialisés, disponibles sur tout support.

C'est dans cette perspective que nous situons notre recherche actuelle. Cependant, pour nous, ce n'est pas tant le lexique final qui compte, que la description de la construction du sens qui émerge dans le processus de conceptualisation. En effet, cela ne nous instruit pas énormément de savoir que l'anglais «software» se dit «logiciel» en français. Ce qui est instructif, par contre, c'est de savoir comment ces mots ont été choisis et pourquoi ils ont retenu l'attention et des terminologues et des utilisateurs qui ont fini par les propager dans la langue générale. Car c'est seulement dans la description analytique de l' histoire de chaque mot, l'évolution de sa forme, les glissements de ses sens, les procédés cognitifs mis en jeu que nous comprendrons comment l'homme, tant individuellement que socialement, $s$ 'approprie de nouvelles connaissances, se construit de nouveaux concepts et, finalement, évolue lui-même en rénovant sa culture.

C'est cette vision que nous allons illustrer dans les trois grandes sections de notre communication. La première partie, consacrée à la construction du sens en néologie terminologique, décrit les aspects linguistiques de cette construction en se référant essentiellement aux exemples du sängö, du wolof et du lingála. La seconde partie traite de l'attitude des locuteurs face à l'innovation, surtout quand celle-ci est massive et fait émerger une langue de spécialité comme un nouveau lieu de pouvoir. Enfï, la troisième partie tente de montrer comment la lexicographie moderne peut aider à résoudre les contradictions et les difficultés naturelles des communautés de locuteurs et d'utilisateurs.

\section{LA CONSTRUCTION DU SENS EN NÉOLOGIE TERMINOLOGIQUE}

En général, quand une technologie nouvelle voit le jour, sa terminologie porte la marque de sa conception. En examinant celle de l'informatique, il est clair que les procédés 
de conceptualisation les plus utilisés sont: la métaphore, la création formelle, l'emprunt, et la normalisation terminologique. Dans les lexiques que nous élaborons dans le cadre de la présente étude, nous avons privilégié ces mêmes procédés, d'autant plus que nous opérons dans un contexte multilingue.

Pour chaque concept à traduire, nous procédons à une analyse approfondie de son sémantisme et, le cas échéant, de ses origines, afin de bien en cerner les contours. Cette démarche permet de mieux repenser le concept dans la langue cible, en fonction de la culture propre de celle-ci. C'est ainsi que s'élabore la construction du sens nouveau qui fonctionnera dans la langue d'arrivée comme l'équivalent conceptuel du terme de départ.

\subsection{LA MÉTAPHORE COMME PROCÉDÉ ESSENTIEL DE CONCEPTUALISATION}

Dans son excellent article sur la métaphore, Assal (1995 : 22) écrit : «En terminologie, la métaphore est à interpréter comme un processus de dénomination et de conceptualisation, en aucun cas comme un procédé d'ornement stylistique; c'est en cela qu'elle se détache de la rhétorique.»

Le domaine de l'informatique est, par sa nouveauté et son évolutivité exponentielle, générateur de terminologies nouvelles très largement métaphoriques. Ainsi les informations, appelées des données, sont consignées dans des fichiers et organisées éventuellement sous forme d'enregistrements. Ceux-ci à leur tour peuvent être organisés en tableaux, en listes chaînées, en piles, en files d'attente, selon la manière dont on veut les exploiter. Les fichiers sont sauvegardés dans des mémoires, et on peut se servir d'une souris pour les afficher dans une fenêtre afin de les lire ou de les éditer. Toutes les informations (données, commandes, adresses, etc.), circulent dans des bus, qui les conduisent à des puces assemblées dans des unités (à l'instar des unités de fabrication ou de traitement dans l'industrie lourde), lesquelles sont, à leur tour, gérées par des pilotes ou des gestionnaires.

La machine à écrire n'est pas l'ancêtre principal de l'ordinateur, mais plutôt les machines à calculer, dont la plus ancienne connue est le boulier. De ce fait, on aurait fort bien pu désigner cette nouvelle machine à calculer qu'est l'ordinateur par le mot «computeur» sans anglicisme, car le verbe «computer» est bien du vieux français et peut donner «computeur» en toute légitimité. Mais on a préféré suivre la proposition faite par Jacques Perret en 1956 de récupérer le mot «ordinateur», libéré de son emploi ecclésiastique. En effet, ce terme désignait, au $\mathrm{XV}^{\mathrm{e}}$ siècle, le prélat chargé de calculer, selon un calendrier lunaire, la date annuelle de la fête de Pâques, par rapport à laquelle toutes les célébrations du cycle liturgique devaient être ordonnancées. Cette fonction n'étant plus du tout pratiquée, le mot «ordinateur», tombé en désuétude, n'était plus employé que dans l'expression théologique «Dieu, grand ordinateur du monde», (cité par Marcellesi 1979: 177). Il pouvait donc sans ambiguité reprendre du service avec un nouveau sens. Comme on le sait, ce fut un succès immédiat.

Ces exemples montrent, s'il en était encore besoin, que les choses nouvelles sont tout naturellement appréhendées par comparaison intuitive avec des choses anciennes déjà connues dans la culture. La différence entre les deux étant faite de traits pertinents que le sujet aura relevés. Comme le fait remarquer très justement Assal : «Pour nommer, quand il est impossible de créer le vocable qu'il faut, la pensée se trouve contrainte à détourner du réservoir de la langue le vocable préexistant qui convient, et qui sera transféré et transposé métaphoriquement pour désigner le concept naissant.» (Assal 1995: 22)

\section{a) Logiciel et matériel}

Lorsqu'on a voulu traduire en français software et hardware, une quinzaine de paires de termes ont été proposées à l'Office de la langue française (OLF), dont mentaille quincaille. Aucune d'elles n'a été retenue. Mais leur étude a permis d'expliciter et d'affiner 
les critères d'acceptabilité dont la prise en compte a permis de trouver le couple logiciel/ matériel qui sera consacré depuis lors.

L'approche américaine considère l'ensemble des programmes nécessaires pour faire marcher un ordinateur comme du «matéricl mou», par analogie avec le «matériel dur» que représente toute la carcasse de la machine. L'approche française a cherché du côté de l'intellect la métaphore de base qui a conduit aussi bien à la proposition de mentaille qu'à celle de logiciel. Tandis que la métaphore de la quincaillerie a servi à créer quincaille qui cédera le pas à matériel, pris, cette fois, dans son sens le plus concret afin de l'opposer à logiciel.

En sängö, la paire de termes qui conviendrait pour rendre ces deux concepts peut être kpëlikua et gbäkürï pour «logiciel» et «matériel» respectivement. Le premier est composé de kpëli «cerveau» et de kua «travail». Le sens littéral du terme kpëlikua est donc «cerveau du travail». Le second désigne les ustensiles, les bagages, les affaires personnelles, donc tout matériel dont on se sert habituellement. Dans le cas d'espèce, il rend bien le concept de «matériel» opposé à «logiciel». En cela, le sängö adopte la même approche que le français. Il se distingue par contre en recourant à la métaphore du «cerveau» qu'il associe au «travail» pour souligner le côté fonctionnel du logiciel. Tout logiciel serait donc vu en sängö comme un produit du cerveau pensé pour réaliser un travail, quel que soit ce travail.

Or, en plus du «logiciel», terme générique opposé à «matériel», il y a lieu de distinguer entre les logiciels «système d'opération» et les «logiciels d'application», une distinction essentielle pour l'utilisateur de l'ordinateur. Les applications sont toujours dédiées à un domaine d'activité bien ciblé comme, par exemple, un traitement de texte, un tableur, un gestionnaire de bases de données, etc. Les applications s'opposent aux systèmes d'exploitation qui, dédiés à l'exploitation des ressources matérielles et logicielles, sont indispensables à toute activité informatisée. On a alors considéré que le concept de kpëlikua, tel que décrit ci-dessus, correspondait mieux à celui de «logiciel d'application». Par opposition, le système d'exploitation a été analysé comme le «cerveau de l'environnement» et appelé kpëlindongoro (littéralement kpëli-cerveau, -ndongoro environnement). On pourrait aussi dire mbandongoro, où $m b a$ - signifie «contrôler, gérer». Le mot mbandongoro signifie donc très exactement «qui gère l'environnement». Pour parler de logiciel en général et notamment par opposition à matériel, on a donc proposé kpëlilö, «cerveau de paroles». Le logiciel est ici vu comme un «cerveau» constitué d'un ensemble de paroles, en l'occurrence, des instructions et des commandes. Le «logos», parole, qui a donné le mot «logique» d'où sera tiré plus tard le terme «logiciel» ne correspond-il pas au lö du sängö? Cette conception converge totalement avec l'importance de la parole agissante dans les traditions culturelles africaines.

En wolof, il a été proposé xelu defin et xelu ndigal pour les concepts respectifs de «logiciel d'opération» et «logiciel d'application», le terme équivalent à «logiciel» étant xelalin opposé à jumtukaay «matériel».

Le sens littéral de xelu defin est «intelligence de la manière de faire, de procéder», tandis que celui de xelu ndigal est «intelligence de l'ordre, de la consigne (sous-entendu une intelligence qui permet d'observer scrupuleusement aussi bien les recommandations que les interdits)».

Les différents logiciels spécifiques sont rendus par des syntagmes complétifs où l'on peut isoler:

1) le radical nominal $x e l$ «intelligence, pensée, esprit, raison»;

2) la marque du connectif au singulier: $u$ «de»;

3) le radical verbal $d e f$ «faire, commettre, exécuter» ou le radical nominal ndigal «ordre, directives, instructions»;

4) les suffixes in et al indiquant respectivement la manière et le causatif. 

intelligent».

Le logiciel est un «instrument qui permet d'acquérir (de) l'intelligence, d'être

Le terme proposé pour matériel est jumtukaay. Ce terme résulte d'une dérivation nominale à partir du substantif jumt servant à désigner tout assemblage d'éléments pour former un tout, voire un objet. Le suffixe $u k a a y$ sert à former des noms d'instruments ou de lieux.

En lingála, les deux termes susceptibles de s'approcher du sens de logiciel et de matériel nous semblent être esálisela et mabendé.

Le premier terme est une dérivation verbale. Il est formé de quatre éléments:

1) le préfixe $e^{-}$, qui indique ici l'objet;

2) le radical verbal -sál- qui signifie «travailler»;

3) les suffixes de dérivation verbale -is- et -il-;

4) la terminaison $-a$.

Vu du lingála, le logiciel serait un «outil qui facilite le travail». Il est essentiel de souligner ici le sens des morphèmes - $i l$ - dit «applicatif» qui exprime bien, nous semblet-il, l'activité de la plupart des logiciels (cf. supra la distinction faite entre «logiciels d'exploitation» et «logiciels d'application») et -is- dit «causatif». Certes, le morphème de dérivation verbale -il-indique, en général, que quelque chose se fait «à la place de», «au bénéfice de» ou «au détriment de» tandis que le morphème -is-traduit l'origine extérieure de l'action, ce qui implique que ni le logiciel ni l'ordinateur ne peuvent agir d'eux-mêmes puisque de toute façon ils ne «remplacent» pas l'utilisateur.

Le second terme proposé est une dérivation nominale, à partir du substantif ebendé signifiant «fer ou tout autre métal». Le préfixe $m a$ - indique, en général, soit un liquide, soit un collectif... «Mabendé» est donc l'ensemble de la ferraille qui compose le matériel informatique (unité centrale et périphériques). «Mabendé» désigne ainsi toute carcasse qui entre dans la constitution de l'informatique. Il se rapproche un peu du mot «quincaille» qui a été écarté au profit de «matériel» en français.

b) Moniteur et écran

En français comme en anglais, on distingue le moniteur de l'écran. Le premier désigne toute la boîte, relativement volumineuse, qui contient l'ampoule cathodique dont le fond phosphaté sert d'écran aux photons. L'écran de l'ordinateur est donc plus précisément cette partie du moniteur sur laquelle on peut voir ce que I'on saisit. Lorsque la technologie a évolué vers les écrans à matrice passive ou active ou les écrans à plasma, la boîte volumineuse du moniteur a disparu et l'on parle d'écran plat, jamais de moniteur plat. Or le terme «moniteur» ne veut pas dire «boîte volumineuse». Son sens étymologique est «celui qui avertit». Le moniteur vous avertit quand vous faites une erreur, et vous permet ainsi de vous corriger et donc de revenir dans le droit chemin. En effet, sans le moniteur, la saisie des données se ferait en aveugle avec toutes les chances de laisser passer beaucoup d'erreurs.

L'écran désignait le panneau servant à se protéger de la chaleur d'un foyer. Selon le Dictionnaire étymologique Larousse (1987), le mot viendrait «sans doute du moyen haut allemand schrank, grille, clôture (allem. mod. signif. «armoire») ou du néerl. scherm, paravent»; le mot était si bien associé à la notion de barrière qu'il a gardé ce sens dans les expressions faire écran, écran de fumée. Dans ces expressions, l'écran est ce qui empêche de voir. Mais avec l'arrivée du cinéma, l'écran n'est plus «la barrière qui empêche de voir», mais «la batrière qui permet de voir». Cette barrière était souvent un mur blanc. De nouvelles expressions ont vu le jour comme mettre à l'écran, être à l'écran, qui ne renvoient plus à une opacité mais bien, au contraire, à l'idée de rendre une ouvre visible par tous grâce au cinéma. Avec la télévision, puis l'informatique, l'écran est devenu la 
page blanche sur laquelle on peut voir ce qu'on écrit ou ce qu'on dessine. On rejoint ici le concept de moniteur analysé ci-dessus.

La description des différents sens et usages dont chaque terme actuel s'est chargé permet de comprendre à la fois les processus de cognition qui ont conduit à l'émergence du sens actuel et les transferts métaphoriques qui ont pu se faire ou ne pas se faire au gré des associations d'images et d'idées. Il devient alors plus clair de voir comment, dans une autre langue et une autre culture, on pourrait procéder pour générer une terminologie acceptable par les locuteurs natifs tout en restant suffisamment précise pour supporter efficacement les concepts du nouveau domaine de connaissance.

En sängö, on prendra plus volontiers en considération la forme volumineuse du moniteur qui rappelle toujours celle d'un pot, du genre «pot de fleurs». Et, puisqu'un moniteur comporte aussi un écran pour afficher et voir ce que l'on saisit, on pourra utiliser soit le mot vulä «exposition, déploiement» (tiré par dérivation tonale du verbe vula «exposer, dévoiler») ou le mot wësë «observation attentive» (tiré par dérivation tonale du verbe wese «observer attentivement») pour créer les mots suivants comme équivalents sängö de «moniteur» en informatique : tavulä, «pot d'exposition», ou tawësë «pot d'observation». On aura sans doute remarqué que tavulä, le pot sur lequel on expose quelque chose, renvoie à un concept plus proche de celui d'écran, tandis que tawësë, le pot qui permet d'observer quelque chose, se rapproche davantage du concept de moniteur.

Mais la métaphore du pot ne tient pas la route lorsque l'écran devient tout plat, tout petit ou, au contraire, géant comme un mur dans une grande salle de cinéma. On dispose en sängö d'autres métaphores appropriées: tatärä «miroir, lunette, verre», kîrä «ardoise de terre cuite» (sur laquelle les écoliers écrivent en classe), sembë «assiette», bâ «emplacement», derêe «mur», etc. Et on peut sans aucune difficulté proposer:

1) pour les écrans plats des portables: tatäräwësë «miroir ou lunette d'observation», ou sembëwësë, sembëvulä «assiette d'observation» et «assiette d'exposition» respectivement; ou encore : kîे ävulä «ardoise d'exposition»;

2) pour un tout petit écran à cristaux liquides, comme sur une calculatrice ou sur le clavier de certains ordinateurs : bâwësë, bâvulä «emplacement d'observation» et «emplacement d'exposition» respectivement;

3) pour un écran géant ou mural : derêvulä, derêwësë «mur d'exposition» et «mur d'observation» respectivement.

Il est incontestable que la pluralité de ces propositions témoigne de la richesse des possibilités créatrices de la langue, mais elle témoigne aussi d'une absence de normalisation en la matière. Et pour cause! Nous sommes ici en néologie absolue! Le sängö de spécialité serait dans ce cas-ci bien plus précis que le français. Mais, dans leur discours quotidien, les locuteurs du sängö qui travailleraient sur ordinateur n'ont pas besoin de plus de précision que ceux des autres langues. Il est donc probable qu'un terme générique les rassure davantage. Dans ce cas, il serait préférable de généraliser un mot court, facile à associer au concept d'écran d'ordinateur, un mot tel que kîrä. Ce terme pourrait désigner l'écran en tant que dispositif matériel, tandis que le terme vulä gardera aisément le sens plus abstrait d'affichage qu'il avait déjà intrinsèquement. On aurait ainsi un couple kîrä et vulä comparable au couple anglais screen et display.

En wolof, la métaphore du berger a été utilisée pour rendre le concept de moniteur Le terme se dit sàmm «berger, pasteur». «Ce qui permet de regarder, d'observer» se dit xoolu. Ce terme nous paraît indiqué pour l'écran de l'ordinateur.

En lingála, nkéni ya bilembo et emśnisi-bilembo est la paire que nous proposons pour rendre compréhensibles les termes de moniteur et d'écran pour les locuteurs lingalaphones. 
«Nkéni» signifie boîte en fer et, par extension, calice. Ce dernier sens se rapproche de «pot» dont l'image entre dans le sens de moniteur pour le sängö (cf. supra). «Bilembo» est le pluriel d' «elembo» qui signifie «figure, signe, symbole, caractère, marque, etc.». Le sens complet du composé «nkéni ya bilembo» est donc «boîte à images, à lettres [d'alphabet]». On retrouve ici tout ce que le moniteur est censé «montrer».

Emónisi-bilembo nous a semblé mieux rendre le sens d'écran. Le premier élément du composé dérive du verbe komónisa «montrer, exhiber, faire paraître, faire voir», lui-même issu du verbe komón. «apercevoir, voir». Le composé entier signifie «le montreur d'images, de caractères, etc.».

\section{c) Clavier et touches}

L'anglais appelle keyboard «planche à clés», et key «clé», ce que le français appelle respectivement clavier et touche. À voir l'objet ainsi dénommé, on imagine mal en quoi il ressemble à une planche à clés. Il y a là de toute évidence des métaphores à l'œuvre. En effet, le mot «clavier» désignait au XII ${ }^{e}$ siècle un «porte-clefs», très probablement un bout de planche sur lequel on accrochait des clés, comme l'atteste le sens non masqué de keyboard. Au XVI ${ }^{\mathrm{e}}$ siècle, ce terme désignait déjà «un assemblage de touches dans divers instruments de musique» (Larousse 1987). Ce nouveau sens était donc déjà résultat d'une métaphore. Mais tandis que les Anglais ont poussé la métaphore jusqu'au bout - ils ont continué d'appeler keys les éléments assemblés correspondant analogiquement aux clés de la planche à clés - , les Français ont préféré privilégier le fait qu'en les touchant, le musicien pouvait jouer de l'instrument en question. Et ils les ont appelées des «touches». Avec l'ordinateur, on a simplement changé de machine (on aurait pu dire d'instrument de musique), mais la métaphore a totalement suivi sans modification.

En Centrafrique, l'instrument de musique traditionnel qui ressemble à une planche, voire à un caisson, et sur lequel un assemblage de touches est arrimé est appelé sanzi. Il porte un autre nom plus ancien et moins répandu, kembî. Les touches de la sanzi sont des lamelles d'acier, de fer, ou de bambou, que l'on fait vibrer avec les pouces. On les appelle

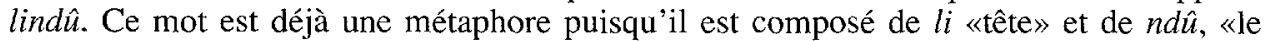
toucher» et signifie donc «la tête du toucher». Il n'y a donc pas de difficulté à appliquer ce même terme lindî aux touches du clavier informatique. Quant au mot clavier, il pourrait être rendu par kembî, mot suffisamment ancien et peu répandu pour ne pas entraîner une confusion avec l'instrument de musique lui-même plus connu sous le nom de sanzi.

En wolof, le clavier est la «planche de l'alphabet», la «planche alphabétique»: lalu araf (littér. lal «lit»u «de» araf «caractère»).

La touche est perçue comme un instrument servant à presser. D'où le terme proposé bësu, composé du radical bës «presser» (un bouton, par exemple) et du suffixe $u$ «instrument».

En lingála, nous avons pensé utiliser également le même instrument de musique qu'en sängö pour nommer le clavier. Cet instrument s'appelle likembé et le terme est sans

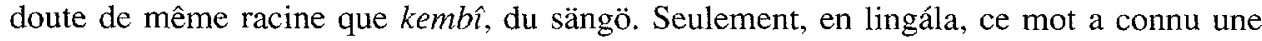
extension de sens dans le vocabulaire de la musique jusqu'à désigner un air de musique ou la guitare que nous avons jugé bon de l'abandonner. Par ailleurs, il existe une variante du mot, moins usitée, ekembé, qui désigne le même instrument et qui pourrait être assigné au clavier.

En gardant la même image de planche de bois (keyboard), nous avons opté pour le mot mángolá pour le clavier en lingála. «Mángolá» est le pluriel de «ngolá» signifiant cauris, ou dés pour jouer. Employé toujours au pluriel, «mángolá» désigne un jeu de stratégie qui se joue à deux sur une planche en bois, appelée elle aussi par métonymie «mángolá». Sur cette planche, sont pratiquées des excavations - en général quatre rangées 
de dix - dans lesquelles on glisse, par paire, soit des cailloux lisses, soit des noyaux de fruits secs que l'on déplace suivant les règles de la variété de jeux.

Quant aux touches de clavier, ce ne sont que des cauris auxquels le mot «mbes€» pluriel de «lobes€» devrait bien convenir.

\section{d) Souris et curseur}

La forme de la souris impose pratiquement cette métaphore dans toutes les langues, semble-t-il. C'est le cas de l'anglais et du français. Par contre, ces deux langues voient le curseur comme un caractère dont la particularité est de courir sur l'écran au fur et à mesure que l'on écrit. Le fait qu'il soit souvent clignotant n'était pas pris en compte. C'était totalement vrai avant l'arrivée de la souris. Depuis, le curseur s'est dédoublé. Une partie, toujours clignotante, indique le point d'insertion actuel. L'autre, indiquant le mode de fonctionnement en cours (insertion de texte, sélection, attente, etc.), ne clignote pas, mais semble glisser à la surface de l'écran quand on déplace la souris.

En sängö, on reprend sans difficulté la métaphore de la souris, qui se dit deko. Par contre, le curseur clignotant est vu comme une luciole qui clignote régulièrement sur l'écran. La métaphore était plus évocatrice du temps des écrans noirs, mais elle reste toujours très suggestive même avec les écrans imitant le papier blanc. On l'appellera donc gepî, «luciole». La partie non clignotante qui semble glisser sur l'écran peut être vue comme un flotteur et est appelée mboyö. Ces métaphores, suggérées par Edema, ici même pour le lingála, conviennent parfaitement à la façon de voir centrafricaine, sans doute parce que les deux langues participent de la même aire culturelle.

En wolof, nos informateurs ont proposé spontanément la métaphore de la souris et de la luciole, en l'occurrence janax et xuyentaan. Il convient de noter que la métaphore pour la partie clignotante du curseur, l'onomatopée xuy-kamaj (litt. «allumer - éteindre») a été écartée au profit de la luciole parce qu'elle évoque le firmament, la voûte céleste, et par analogie l'écran de l'ordinateur. Cependant xuy-kamaj permettra de nommer le voyant (tout signal lumineux destiné à avertir l'utilisateur).

En lingála, on gardera aussi la métaphore de la souris, mpúku, comme en anglais et en français, pour indiquer ce prolongement de la main. Cependant, deux mots ont été proposés pour traduire le curseur: motúmbe et língóngongó. Motúmbe désigne, en lingála, le flotteur de pêche. Il a pour synonyme litukú et likombo. Mais comme ces derniers termes sont des noms d'espèces d'arbres dont on fait le motúmbe, nous l'avons privilégié au détriment des litukú et likombo. De plus, il n'a que ce sens-là. La partie fixe sera nommée ici motúmbe et la partie clignotante língóngongó. Le curseur fixe n'est-il pas semblable à un morceau de bois qui flotte sur l'écran ? Et comme la partie clignotante scintille de façon régulière dans la «nuit» de l'écran, la luciole peut légitimement entrer dans l'informatique, à l'instar de la souris et de la puce.

Laissons à Assal le dernier mot sur ce point: «La métaphorisation terminologique est une nécessité inhérente de la pensée scientifique, elle est sa dynamique interne. Lorsqu'un savant, un chercheur emprunte des termes en raison de leur commodité allusive, de leur pouvoir évocateur, ce n'est pas seulement par souci de se faire comprendre, ce n'est pas seulement une question de terminologie, c'est fondamentalement une affaire de conceptualisation.» (Assal 1995: 22)

\subsection{LA CRÉATION LEXICALE FORMELLE}

La vitalité d'une langue est souvent rapportée à sa capacité de générer de nouveaux mots immédiatement acceptables par les locuteurs, parce que conformes à des règles de formation reconnues dans la langue. Chaque famille de langues, voire chaque langue, a 
ses propres règles de formation des mots, quand bien même elles peuvent être ramenées aux grands types classiques que sont la dérivation, la composition, la siglaison, la flexion, la troncation, etc. Nous n'allons pas détailler ici tous les procédés de création formelle dont dispose le terminologue, mais seulement illustrer ceux qui, en sängö, en wolof et en lingála, entrent dans la création des mots qui nous concernent.

En sängö, il existe plus d'une douzaine de schèmes de composition nominale qui sont très productifs (cf. Diki-Kidiri 1977: 86-115). Tous les noms composés présentés ici relèvent de ces schèmes. La dérivation est davantage utilisée dans la formation des verbes et des nominaux qui en dérivent (cf. Diki-Kidiri 1977: 72-80). Trois des dérivatifs méritent d'être rappelés à notre attention, car ils sont particulièrement intéressants pour le travail en cours: la dérivation tonale, les suffixes -nga et -ngbi.

La dérivation tonale consiste ici en l'élévation du ton final d'un verbe au registre supérieur. Les autres syllabes du radical verbal peuvent ou non subir des changements sans conséquences. Le dérivé ainsi formé peut désigner un organe, le résultat du procès référé par le verbe, une propriété. Elle est donc utilisée dans la création de termes comme sungä «un enregistrement», de sînga «enregistrer»; vulä «affichage, exposition», de vula «afficher, exposer», etc.

Le suffixe -nga ajoute au radical verbal l'idée d'une activité codifiée, quelque chose qui s'accomplirait selon un protocole donné, une périodicité régulière, ou un schéma précis. On peut dire, par exemple, qu'enregistrer un fichier, ce n'est pas seulement l'écrire, mais l'écrire dans un registre; ce qui implique un protocole, un format dans lequel le fichier sera transcrit afin d'être récupérable. Donc nous dirons sûnga pour «enregistrer», néologisme dérivé de $s \hat{u}$ «écrire».

Le suffixe -ngbi implique plusieurs actants dans le procès, soit qu'il y ait plusieurs sujets agissant ensemble, soit qu'il résulte du procès plusieurs objets. Ainsi, $k \hat{a}$ signifie «être fêlé», kângbi «diviser, partager». Ce suffixe étant très productif en sängö, son application dans la formation de nouveaux concepts ne pose aucun problème. Ainsi, à partir de sîo «planter (un pieu en terre)» on a proposé sîôngbi pour «configurer», car il s'agit là aussi de planter le décor, en positionnant plusieurs paramètres à la fois comme autant de pieux.

En wolof, le procédé de création lexicale le plus utilisé est, d'une part, la dérivation par affixation, réduplication ou alternance consonantique et, d'autre part, la composition. Mais il existe également des procédés syntaxiques (Dumont et Mbodj 1978: 449-467; Mbodj 1994: 4).

En ce qui concerne la dérivation nominale, les suffixes kat et $u$ (kaay) permettent de former respectivement des noms d'agents et des noms d'instruments ou de lieux à partir de verbes. Ces suffixes sont fréquemment utilisés, notamment par les chercheurs qui souhaiteraient que la terminologie de l'informatique ne soit pas opaque et que, par conséquent, on fasse moins appel à la métaphore qu'à la création formelle à partir de verbes, de substantifs ou autres dont le sens est connu par les jeunes générations, fût-ce un mot emprunté au français. Il s'agira certainement d'équilibrer la métaphore terminologique avec la création formelle.

On trouve également dans notre corpus, consacré au vocabulaire de l'informatique, le suffixe aay «relatif à ce qui est...» susceptible de produire des noms «abstraits», taxawaay «format (d'impression)» à partir de taxaw «se mettre debout, être debout».

En lingála, comme dans la plupart des langues bantoues, la morphologie est très riche. Ce qui permet plusieurs formes de création lexicale soit à partir de nominaux et adjectivaux, soit à partir de verbaux.

Deux mécanismes de création sont possibles en lingála: la dérivation simple ou complexe et la composition. 


\section{a) La dérivation simple}

Cette dérivation fonctionne sur la base du système d'affixes (préfixes et suffixes) qui s'ajoutent au radical nominal ou verbal mais qu'il serait trop long de présenter en totalité ici.

La structure des dérivés nominaux est composée de deux éléments: le préfixe nominal et le thème nominal. Dans la dérivation nominale, seul le préfixe peut changer pour donner lieu à un autre substantif. En substituant au préfixe $e$ - de «ebendé» (fer, dont le pluriel est bibendé) le préfixe ma-, nous pouvons créer un néologisme «mabendé» pour signifier ferraille et, par métaphore, arriver à nommer «matériel».

La formule de la dérivation verbale, elle, est composée de trois éléments : le préfixe nominal, le thème déverbatif et le suffixe. Ici, deux éléments sur trois peuvent commuter avec d'autres pour former un nouveau dérivé à partir d'une même base verbale. Ainsi, en partant de la locution verbale du lingála «kobéte masíni» qui signifie «dactylographier» (littéralement «frapper la machine [à écrire]»), nous aurions pu forger un nouveau mot, «ebételo », pour dire clavier (lieu où l'on «tape», où l'on dactylographie), et prendre

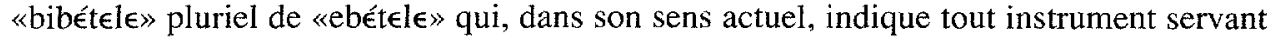
à frapper, ou à enfoncer (marteau, par exemple), pour nommer les touches du clavier, «ebételob. On voit bien dans cette paire que seuls les suffixes $-\dot{\partial}$ et $-\epsilon$ changent, le reste des éléments demeurant inchangés. Il faut signaler que le mot «ebételi» existe en lingála et signifie «manière, façon de frapper, de jouer (un instrument de musique, par exemple)».

Sans que cela passe pour une règle absolue ou une loi, on peut toutefois souligner la valeur sémantique des préfixes et des suffixes. Ce sémantisme n'est qu'une tendance. Nous laissons de côté les préfixes, même si leur choix dans la dérivation n'est pas arbitraire. Qu'il suffise de noter que le choix des suffixes est moins aléatoire que celui des préfixes dans la dérivation.

Quatre suffixes interviennent dans la formation des nominaux à partir des verbes:

1) le suffixe -á ou -a; il exprime en général soit une action ou une activité : mokúmbá bagage, soit un résultat libóta famille, descendance;

2) le suffixe $-i$; il exprime ce qui se fait habituellement, la façon, la manière de faire; elakisi signifiera ainsi ce qui indique, ce qui montre habituellement (l'écran);

3) le suffixe -o exprime le lieu où s'exécute une tâche; ainsi ebómbelo, «lieu où I'on cache», magasin, dépôt, grange, cave, réservoir, nommerait bien le disque dur;

4) le suffixe - $e$ indique l'instrument qui sert à; ekomele (tout instrument pour écrire; stylo, bic, touche, plume) pourra servir à nommer l'imprimante.

\section{b) La dérivation complexe}

Cette dérivation est dite complexe non seulement parce qu'elle procède souvent par le redoublement du thème verbal mais aussi et surtout parce qu'il s'y ajoute un changement de ton dans la base. La deuxième partie du dérivé n'est généralement pas du même schème tonal que la première.

\section{c) La composition}

La composition se fait par juxtaposition des nominaux (avec ou sans fonctionnel) ou d'un substantif dérivé du verbe et d'un nominal. Nous avons ainsi nkéni ya bilembo, «boîte à images» pour moniteur et elakisi-bilembo, «ce qui montre les figures, les caractères» pour l'écran. 


\subsection{L'EMPRUNT SÉLECTIF COMME PROCÉDÉ D'INNOVATION CONCEPTUELLE} Comme le fait remarquer Zygmunt Stoberski (1987: 88):

...the development of a language depends not only on its own inner laws, as is the case with an individual, a family and a nation, but on external influences, contacts, and on adapting foreign words as well.

L'emprunt est donc une nécessité vitale, à condition, toutefois, qu'il soit fait à dose homéopathique. Il convient de hiérarchiser les langues sources. On empruntera par ordre de priorité :

1) aux langues les plus apparentées à la langue cible: langue mère, puis langues sœurs, etc.;

2) aux langues importantes en contact avec la langue cible, sauf là où il importe de protéger l'identité de la langue cible;

3) aux langues étrangères par lesquelles la technologie nouvelle est arrivée.

En sängö, l'application de ces principes donne une priorité absolue au ngbandi, langue mère du sängö, puis, dans l'ordre, les autres langues centrafricaines, les langues africaines en contact (dont le lingála), le français et, finalement, les autres langues étrangères ayant cours dans la région ou qui présentent un intérêt pour la terminologie en sängö.

Le sängö a emprunté au français dokimäa «document», finêtere «fenêtre» que nous proposons de garder en informatique. Dans le cas de finêtere, on aurait sans doute mieux fait d'emprunter à l'anglais «window» adapté en wîndo et qui serait uniquement utilisé en informatique. En effet, dans certains cas, l'anglais donne des emprunts bien plus facilement adaptables en sängö que le français. Ainsi, *orodinatëre n'aurait pas pu se maintenir devant kombûta de l'anglais «computer» pour «ordinateur».

Les emprunts ont parfois une histoire qu'il est bon de connaître. Au début des années 1900 , le terme «döpulä» désignait la fenêtre des petits kiosques où l'on vendait un peu de tout. C'est par cette fenêtre que le vendeur et l'acheteur échangeaient marchandise et monnaie. D'où l'appellation «dö-pulä» littéralement «trou (de) commerce». Avec le développement des magasins et boutiques plus importantes où les clients pouvaient accéder directement au comptoir à l'intérieur de l'immeuble, les kiosques ont perdu de leur importance et le terme est tombé en désuétude. Il est de temps en temps utilisé par certains locuteurs au sens de «fenêtre», concurremment à l'emprunt au français «finêtere». Aujourd'hui, avec la réapparition des kiosques à journaux et des kiosques à distribution de produits alimentaires (boissons, beignets, pain, sandwiches, etc.) le terme retrouve son emploi originel qui rend bien le concept de «kiosque». En informatique, il faudrait sans doute mieux préférer wîndo, ou à la rigueur finêtere pour «fenêtre».

L'emprunt peut entrer partiellement dans un mot-valise comme dans kombâkîrä

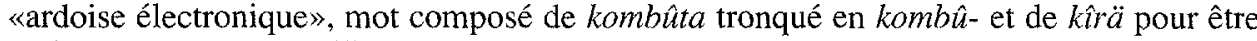
moins long et plus équilibré euphoniquement. Ce qui lui donne une meilleure chance de s'intégrer rapidement au sängö.

En wolof on pourrait aussi appliquer les mêmes principes qui régissent l'emprunt en sängö à ceci près que, généralement, le wolof emprunte à l'arabe, le plus souvent de façon inconsciente; certains mots d'origine arabe sont tellement intégrés au wolof qu'il faudrait être un érudit de l'arabe pour pouvoir les détecter. Ainsi en est-il de araf, «caractère». Mais l'emprunt peut aussi se faire de façon consciente comme on l'a fait au français pour les mots disk, «disque» et pom «pomme» (de Macintosh).

L'emprunt résulte du souci de préciser le lexique, en évitant de recourir à la périphrase qui ne serait d'aucune façon plus explicite. L'emprunt obéit ici en quelque sorte à un double principe d'économie et d'enrichissement de la langue. 
En lingála l'emprunt se fait aussi bien aux langues locales (kikongo, swahili, etc.) qu'aux langues européennes (anglais, français, portugais). Naturellement, selon les principes retenus ci-dessus, ce sont les premières qui seront privilégiées dans l'emprunt parce qu'elles sont linguistiquement (étant de la même famille) et culturellement proches du lingála. En examinant attentivement le lexique du lingála, on constatera qu'un mot comme lininisa «fenêtre» est un emprunt au swahili dirisha. Ce mot traduit bien l'anglais «window».

\subsection{LA NORMALISATION DES CHOIX NÉOLOGIQUES EN TERMINOLOGIE}

Certaines notions sont si proches que leur mise en concept peut se faire de différentes façons, donnant ainsi des possibilités de choix entre des dénominations différentes. Il importe, dans ce cas, que, par une opération de normalisation, chaque nuance conceptuelle se voie attribuer une dénomination unique. Ainsi, on peut dire qu'«enregistrer» un fichier, c'est aussi le «sauvegarder», mais conceptuellement, il s'agit de notions distinctes.

En sängö, il y a plusieurs façons de dire «ouvrir», alors comment dira-t-on «ouvrir un fichier»? Le concept d'ouvrir couvre plusieurs conceptions en sängö : «fungûla» correspond plus exactement à «ouvrir un espace clos généralement avec une clé», soit «déverrouiller», mais on dira plutôt «zî yâ tî...» (littéralement: «enlever intérieur de...») pour «ouvrir un livre» car on le fait en écartant les pages du livre pour en exposer l'intérieur. Si «ouvrir» implique l'enlèvement d'un couvercle, on dira plutôt «zî yângâ tî...», enlever l'ouverture de. Dans le cas d'un fichier informatique, «ouvrir» est une métaphore qui correspond à «donner l'ordre de copier le fichier en mémoire centrale et de l'afficher sur l'écran». Étant donné que toute opération d'affichage implique nécessairement que le fichier à afficher ait été préalablement copié en mémoire centrale, l'ordre «d'ouvrir un fichier» peut être considéré comme équivalent de celui d'afficher un fichier. On peut dans ce cas utiliser le verbe «vula» qui veut dire «dévoiler, afficher, exposer» pour rendre «ouvrir». Mais si l'on préfère distinguer les deux notions «ouvrir» et «afficher», alors on réservera «vula» pour «afficher», et l'on devra choisir un autre terme pour «ouvrir». On peut considérer qu'ouvrir un fichier c'est l'appeler pour l'afficher. On le rendra alors par «îri», appeler. Tant que l'utilisateur peut aisément comprendre «îri» avec le sens d'«appeler», l'expression est parfaitement viable en sängö. Mais le verbe «îri» peut aussi signifier «nommer» et, dès lors, il y a une possibilité qu'un locuteur sélectionne le mauvais sens et comprenne «nommer un fichier» là où il fallait comprendre «appeler un fichier». Il serait donc préférable d'utiliser «fungûla». Seulement, «fungûla», hérité du kiswahili a pris, en sängö, le sens d'«ouvrir une porte qui a été fermée à clef, déverrouiller». Il conviendrait donc mieux à exprimer la notion de «déprotéger (un fichier)» en informatique. En définitive, nous proposons le verbe ngbändï «dakpa» qui veut dire «ouvrir (la bouche)».

En wolof, on peut signaler, en guise d'exemple, que deux termes ont été proposés pour éjecter (une disquette) : génne «faire sortir, expulser, chasser» et rocci «retirer un objet enfoncé». La difficulté réside ici dans le fait que, par rapport à la langue générale, génne supposerait que c'est l'ordinateur qui agit (ce qui n'est pas loin de la vérité si l'on s'en tenait au sens étymologique du mot ordinateur «celui qui»), alors que rocci indiquerait que c'est l'utilisateur qui doit agir. Rocci évoque en outre l'idée de fente. C'est pourquoi il a l'avantage sur génne.

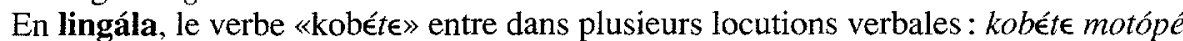

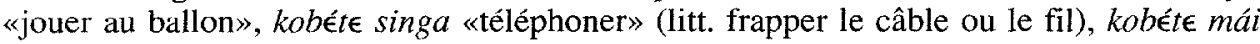

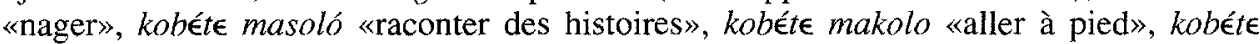
masíni «dactylographier», etc. Le dérivé nominal «ebételj» qui en découle signifierait littéralement «lieu où il se frappe quelque chose». De ce fait, il peut désigner une grande 
diversité de choses telles que: un stade de football, un pool de dactylographie, une imprimerie, une piscine (où l'on «bat» en nageant), un standard téléphonique, etc. Dans un domaine technique, un terme a toujours un sens univoque, quelle que soit la charge polysémique qui est la sienne dans la langue générale. Toutefois, il nous semble préférable de recourir, chaque fois que cela est possible, à des étymons moins polysémiques pour créer des néologismes.

\section{DE LA LANGUE GÉNÉRALE AUX LANGUES DE SPÉCIALITÉ}

L'innovation terminologique se différencie de l'innovation lexicale dans la langue générale par le fait qu'elle est destinée à l'usage de professionnels. D'où la formation de langues de spécialité, comprises uniquement des spécialistes. Les autres, les non spécialistes du domaine, vivent ces langues-là comme des «jargons» de spécialistes. En général, les langues de spécialité déteignent progressivement sur la langue générale au fur et à mesure que les produits technologiques ou les centres d'intérêt des spécialistes deviennent populaires, et tout le monde finit par en entendre parler.

\subsection{TERMINOLOGIE ETT PHRASÉOLOGIE}

Même quand tous les mots techniques sont tirés du langage commun par métaphorisation, le discours technique peut rester totalement incohérent pour un non initié. Sans un minimum de culture informatique, un locuteur français trouverait complètement aberrant un ordre comme celui-ci: «Ouvrez une fenêtre avec votre souris et chargez-y le fichier autoexec.bat». Au premier degré, une fenêtre s'ouvre avec une poignée, pas avec une souris! Et puis, un fichier, c'est en général une boîte remplie de fiches en carton, comment peut-on le charger dans une fenêtre?

Il est donc évident que même si nous traduisons dans les langues africaines la terminologie informatique pour l'utilisateur de l'ordinateur, il faut encore que celui-ci acquière un minimum de culture informatique. Heureusement, cela se fait, le plus souvent, en travaillant avec l'ordinateur, c'est-à-dire dans le concret de l'action.

\subsection{LES ATTITUDES DES LOCUTEURS FACE À LA NÉOLOGIE TERMINOLOGIQUE}

Il n'est pas rare de voir des locuteurs ayant une haute idée de leur langue affirmer avec conviction que celle-ci peut tout exprimer, et cependant rejeter globalement toute innovation que d'autres locuteurs y apportent. Cette attitude contradictoire s'explique par une confusion entre langue générale et langues de spécialité. Ce qui est le cas de la plupart des gens dans une société où la spécialisation des connaissances et des professions n'est pas très poussée. Cependant, une langue de spécialité est d'abord destinée à l'usage des spécialistes d'un domaine donné. Lorsque ce domaine commence à acquérir de la lisibilité dans la communauté linguistique générale et que les spécialistes de ce domaine commencent à être identifiés en tant que groupe social, la langue de spécialité est acceptée comme telle. Dès lors, une partie de la terminologie développée dans ce domaine se répand progressivement dans la langue générale. Les attitudes sont donc sujettes à des variations qui méritent une observation sur la durée.

\section{LES SOLUTIONS LEXICOGRAPHIQUES}

On peut améliorer l'implantation de la terminologie nouvelle d'une part, en veillant à ce que, linguistiquement, les termes retenus ne soient pas des périphrases plus ou moins raccourcies, ni ne produisent, par leur fréquence ou cooccurrence, une succession de mots rares d'autre part, en formant les utilisateurs de l'ordinateur dans cette terminologie dès les premiers pas. 
Des lexiques terminologiques seront toujours nécessaires, sous les formes les plus adaptées à chaque situation : formation initiale, formation avancée, assistance professionnelle. Le choix entre support papier et support électronique dépend directement des contextes d'utilisation. En Afrique, où l'ordinateur est encore un instrument des plus rares, le contexte le plus dominant requiert des lexiques sur support papier.

\subsection{LE LEXIQUE SUR SUPPORT PAPIER}

En Afrique, les ouvrages sous forme traditionnelle de livres en papier sont incontestablement les plus pratiques à utiliser dans toutes les situations. Ils sont toujours accessibles sans aucun autre accessoire. Il est donc important, dans le cas de notre recherche actuelle, de produire des lexiques de ce type quand bien même d'autres formes de lexiques peuvent être envisagées.

\subsection{LE LEXIQUE ÉLECTRONIQUE MULTIMÉDIA ET HYPERTEXTUEL}

Dans le cadre d'un poste de travail informatique, il est avantageux de disposer d'un didacticiel multimédia incorporant les lexiques terminologiques élaborés à l'issue de cette recherche. En effet, il est bien plus efficace d'apprendre à quelqu'un à se servir de l'ordinateur, si non seulement on le fait dans sa langue, mais encore si le locuteur peut, à volonté, écouter chaque mot, chaque explication, les lire à l'écran et les voir en action dans des images animées.

\section{EN CONCLUSION}

Un travail comme celui-ci représente une sorte de test en laboratoire pour observer l'impact de la néologie terminologique sur les sociétés humaines en général et africaines en particulier. On peut y étudier notamment les processus d'acquisition des connaissances nouvelles, le traitement de l'altérité par l'ordre cognitif établi, les comportements humains face à l'inconnu que représentent les domaines spécialisés, enfin les approches lexicographiques susceptibles d'apporter des solutions concrètes à des difficultés concrètes bien décrites.

Pour aller encore plus loin dans cette discussion, il y aurait lieu de se pencher sur la question de l'harmonisation internationale de la terminologie. Les terminologies que nous pouvons élaborer en sängö, en lingála, en wolof, ou dans d'autres langues africaines, même dans un cadre aussi restreint que celui-ci, où elles sont à visée utilitaire, participent de la terminologie générale de l'informatique. Dès lors, on peut se demander quels seraient les choix théoriques si l'on devait développer un enseignement général et avancé de l'informatique dans ces langues. Nous laisserons les lecteurs sur cette interrogation.

\section{Note}

* Cet article est issu d'une communication présentée par l'auteur aux IV ${ }^{e s}$ Journées scientifiques du réseau «Lexicologie, terminologie, traduction» de l'AUPELF-UREF (Lyon, France, 28, 29, 30 septembre 1995).

\section{RÉFÉRENCES}

ASSAL, A. (1995): «La métaphorisation terminologique», L'actualité terminologique - Terminology Update, 28 (2), ministère des Travaux publics et Services gouvernementaux Canada, pp. 22-24.

BOUQUIAUX, L. et al. (1978) : Dictionnaire sango-français - Lexique français-sango, Paris, SELAF, coll. «TO», $\mathrm{n}^{\circ} 29,600 \mathrm{p}$.

CELTA (1974): Actes du I ${ }^{e r}$ Séminaire des linguistes du Zaïre, Lubumbashi, $301 \mathrm{p}$.

DAUZAT, A et al. (1987): Dictionnaire étymologique et historique, Paris, Larousse.

DIKI, KIDIRI, M. (1977) : Le sängo s'écrit aussi..., Paris, SELAF, coll. «TO», n 24, $187 \mathrm{p}$.

DIOP, C. A. (1975): «Comment enraciner la science en Afrique: exemple wolof (Sénégal)», Bulletin de l'Institut fondamental d'Afrique, série $\mathrm{B}$, tome $37, \mathrm{n}^{\circ} 1$, pp. 154-233. 
DUMONT, P. et C. MBODJ (1978): «Le wolof, langue de développement: étude des procédés d'enrichissement de la langue wolof», C. Hagège et I. Fodor (dir.), La réforme des langues, histoire et avenir, vol. 1 , pp. 449-461.

MARCELLESI, C. (1979) : «Retour aux sources: quelques aspects du vocabulaire de l'informatique», Adda et al., Néologie et lexicologie, Paris, Larousse, coll. «langue et langage», pp. 176-183.

MBODJ, C. (1994): «L'activité terminologique au Sénégal», Terminologies nouvelles, 11, ACCT et Communauté française de Belgique.

SAULNIER, P. (1994) : Lexique orthographique sängö, Bangui, Centre pastoral, 48 p.

STOBERSKI, Z. (1987) : «Transnationalization of Terminology», E. Dagne et D. Geneda (Eds), Proceedings of the International Seminar on Terminology, january 16-18, 1986. Addis-Ababa, Ethiopia, The Academy of Ethiopian Languages Science and Terminology Terms Translation Project, pp. 87-89.

Annexes

Nous ne présentons dans ces annexes que quelques extraits de vocabulaires, esquissant les lexiques actuellement en cours d'élaboration.

\section{A. Extrait du lexique informatique français-sängö}

\section{Aspects matériels}

Aspects extérieurs de la machine

ordinateur : kombûta

boîtier (en métal) : kopu

clavier : kembî

écran mural (de projection) : derêvulä

écran plat : kîrä

imprimante : sasango

moniteur : tavulä

souris : deko

touche : lindû

\section{Aspects internes de la machine}

microprocesseur : sambûta

bus : balabâla

carte : kârâte

contrôleur : wambängö

contrôleur d'écran : wambängö-kîrä

\section{Performances}

mémoire : go

mémoire morte : mîtâ go

mémoire vive : yepere go

mémoire tampon : gohâko

mémoire cachée : gohônde

mémoire centrale : gokua

mémoire virtuelle : gombïngo

\section{Logiciels}

logiciel (génér.) : kpëlilö

logiciel d'application : kpëlikua

logiciel de dessin : kpëlilimo

logiciel principal (dans un système) : tökpëliłö

système d'exploitation: mbandongoro

système d'opération ou système de gestion

d'environnement : $k$ pëlindongoro

tableur : kpëlikpäkpä

\section{Activités}

affichage, ce qui est affiché : vulä

afficher : vula

copier : sûngi couper (une sélection) : fângbi

curseur : gepî

déplacer: dônzi

désactiver : mîngo

déverrouiller, déprotéger : fungûla

document : dokimäa

document (nouveau -) : finî dokimäa

donnée : kôngbâ

donnée brute : kukûu kôngbâ

donnée traitée : lêkua

enregistrer : sunga

enregistrement (action d' enregistrer quelque chose): süngängö

enregistrement (ce qui est enregistré) : sungä

enregistrer sous... : sunga töngana..

enregistrer tout : sunga kûêt

établir: senga

fenêtre : finêtere

fermer un fichier : kânga

fichier: kuru

imprimer: pete

insérer : yôro

installer: senga

instruction, commande : mbëlä

nommer (un fichier) : îri

outil : yêkua

ouvrir un fichier : dakpa

quitter : sîgî

renommer : sanzêe ïrì

répertoire : kanzalindo

saisir à l'ordinateur : mbûta

sauver, sauvegarder : sôo

sélectionner, trier : soro

supprimer: lungûla

\section{B. Extrait du lexique informatique français-wolof}

\section{Aspects matériels}

Aspects extérieurs de la machine

ordinateur : jumtukaayu xel

clavier : lalu araf

touche : bësu

souris : janax

écran (moniteur) : xoolu 
imprimante : moolukaay

câble (- fiche) : nsinga

lecteur: jàngukaay

disque dur: diskbi

disquette : ndisksi

encre : daa

Activités

aide : ndimma

caractère : araf

colonne : jën

commandes : santaane

copier: sotti

curseur : xuentaan

effacer : far

éjecter : rocci

entrer: dugg

fenêtre: palanteer

fermer un fichier : ub ag kàggu

fichier : kàggu

imprimer : mool

menu: sas

ouvrir un fichier : ubbi ag kàggu

quitter : dëdd

sauvegarder : rawale

sortir : génn

C. Extrait du lexique informatique français-lingála

\section{Aspects matériels}

Aspects extérieurs de la machine

ordinateur: esálela

clavier : mángolá, gbagba, ekembé

touche : molembo, túsi

souris : mpúku, mpô

écran (moniteur) : emónis imprimante : ebimiselo

câble (- fiche) : nsinga

pavé numérique : etando ya mitúya

lecteur : etángela

disque dur : ebómbelo enéne, (dísiki -)

disquette : ebómbelo iké, (dísiki -)

CD-ROM : paláki

haut-parleur: nsábí, losébá

périphérique : zíngá-zíngá

gomme : ezimeli (gúmi)

\section{Aspects internes}

microprocesseur : bongó

Performances

mémoire morte : etando

\section{Activités}

aide : bosálisi

caractère : elembo

commandes : etámboseli

copier : koákisa

curseur : motúmbe, língóngongó

effacer: kozíma

éjecter : kobimisa

entrer : kokótisa

fenêtre : lininísa

fermer un fichier : kokanga

fichier : kásá-kásá

formater une disquette : kobongisa ebúkútu

informatique : bonganga masíni

ouvrir un fichier : kofungola kásá-kásá

quitter : kobima

racine : lintína

repertoire : etápe

saisir : kobéte

sauvegarder : kobíkisa

sortir : kobima 